\title{
A!
}

This is an electronic reprint of the original article.

This reprint may differ from the original in pagination and typographic detail.

Salminen, Nelli H.

\section{Human cortical sensitivity to interaural level differences in low- and high-frequency sounds}

Published in:

Journal of the Acoustical Society of America

DOI:

$10.1121 / 1.4907736$

Published: 01/01/2015

Document Version

Publisher's PDF, also known as Version of record

Please cite the original version:

Salminen, N. H. (2015). Human cortical sensitivity to interaural level differences in low- and high-frequency sounds. Journal of the Acoustical Society of America, 137(2), EL190-EL193. https://doi.org/10.1121/1.4907736

This material is protected by copyright and other intellectual property rights, and duplication or sale of all or part of any of the repository collections is not permitted, except that material may be duplicated by you for your research use or educational purposes in electronic or print form. You must obtain permission for any other use. Electronic or print copies may not be offered, whether for sale or otherwise to anyone who is not an authorised user. 


\section{Human cortical sensitivity to interaural level differences in low- and high-frequency sounds}

Nelli H. Salminen

Citation: The Journal of the Acoustical Society of America 137, EL190 (2015); doi: 10.1121/1.4907736

View online: https://doi.org/10.1121/1.4907736

View Table of Contents: http://asa.scitation.org/toc/jas/137/2

Published by the Acoustical Society of America

\section{Articles you may be interested in}

The dominant role of low-frequency interaural time differences in sound localization

The Journal of the Acoustical Society of America 91, 1648 (1992); 10.1121/1.402445

Evaluation of a method for enhancing interaural level differences at low frequencies

The Journal of the Acoustical Society of America 140, 2817 (2016); 10.1121/1.4965299

The influence of pause, attack, and decay duration of the ongoing envelope on sound lateralization

The Journal of the Acoustical Society of America 137, EL137 (2015); 10.1121/1.4905891

Perception of across-frequency interaural level differences

The Journal of the Acoustical Society of America 122, 2826 (2007); 10.1121/1.2783130

Discrimination of interaural differences of level as a function of frequency

The Journal of the Acoustical Society of America 83, 1846 (1988); 10.1121/1.396520

Interaural level difference processing as a function of frequency

The Journal of the Acoustical Society of America 140, 3101 (2016); 10.1121/1.4969676 


\title{
Human cortical sensitivity to interaural level differences in low- and high-frequency sounds
}

\author{
Nelli H. Salminen \\ Brain and Mind Laboratory, Department of Biomedical Engineering \\ and Computational Science and MEG Core, Aalto NeuroImaging, \\ Aalto University School of Science, Espoo, Finland \\ nelli.salminen@aalto.fi
}

\begin{abstract}
Interaural level difference (ILD) is used as a cue in horizontal sound source localization. In free field, the magnitude of ILD depends on frequency: it is more prominent at high than low frequencies. Here, a magnetoencephalography experiment was conducted to test whether the sensitivity of the human auditory cortex to ILD is also frequency-dependent. Robust cortical sensitivity to ILD was found that could not be explained by monaural level effects, but this sensitivity did not differ between low- and high-frequency stimuli. This is consistent with previous psychoacoustical investigations showing that performance in ILD discrimination is not dependent on frequency.

(C) 2015 Acoustical Society of America

[QJF]

Date Received: December 10, 2014 Date Accepted: January 25, 2015
\end{abstract}

\section{Introduction}

Human sound source localization in the horizontal plane relies on binaural cues: the interaural level and time difference (ILD and ITD, respectively). ${ }^{1}$ The relative importance of the two cues depends on sound frequency. ITD is best detected from the finestructure of low-frequency sounds ${ }^{1}$ while ILD is more prominent at high than at low frequencies. ${ }^{2}$ However, the importance of ILD specifically in high-frequency sounds is not fully reflected in the perception of ILD in head-phone listening. The detection thresholds for ILD are only weakly dependent on sound frequency. ${ }^{3,4}$

Previous studies have shown that the human cortex is sensitive to ILD (Refs. 5 and 6) but the dependency of this sensitivity on sound frequency remains unexplored. Therefore, the present study tested whether there is neural specialization to ILD at high sound frequencies where it is more relevant for sound source localization. To this end, the sensitivity of the human auditory cortex to ILD in low- and high-frequency sounds was recorded in magnetoencephalography (MEG). An important challenge in brain studies of ILD processing is to distinguish between sensitivity to binaural cues and potential effects of monaural sound level. To avoid this confound, the sound presentation was designed to produce opposing predictions for genuine ILD sensitivity and for monaural level effects.

\section{Methods}

Twelve healthy volunteers took part in the experiments ( 2 males, age mean 24 , standard deviation 4) with written informed consent and the approval of the Ethical Committee of Aalto University. The data of one participant were discarded due to excessive movement artifacts. During the recordings, the participants were instructed to ignore the sound stimulation and to focus on reading a self-selected text.

The stimuli were 200-ms bursts of bandpass noise with 10-ms onset and offset ramps. The noise bands were three equivalent rectangular bandwidths wide and centered at $500 \mathrm{~Hz}$ (low-frequency stimulus) or $4 \mathrm{kHz}$ (high-frequency stimulus). The sounds were presented through an MEG-compatible tube-phone system with insert tips. At 0 ILD the sound level was set at $65 \mathrm{~dB}$ (sound pressure level $A$ ) for both 
stimulus frequencies. ILD was introduced by incrementing the sound level in one ear and decreasing it by the same amount in the other. Ten independent samples of each stimulus were used. The sounds were presented following a stimulus-specific adaptation paradigm [Fig. 1(A)]. The sound presentation alternated between a probe and an adaptor sound with a stimulus onset asynchrony of $1 \mathrm{~s}$ (i.e., a probe occurred every $2 \mathrm{~s}$ ). In general, response amplitudes are larger when the adaptor and probe locations differ than when they are the same. ${ }^{7}$ Each probe-adaptor pair was presented in a separate stimulation block and the presentation order of the blocks was randomized for each subject.

Two probe sounds were used for each frequency: +10 and $+30 \mathrm{~dB}$ ILD [right ear louder; Fig. 1(B)]. The $+10 \mathrm{~dB}$ probe sound was presented with adaptors of +10 and $-10 \mathrm{~dB}$ (left ear louder) ILD. The relatively large ILD difference was found necessary during pilot experiments for revealing robust ILD sensitivity. This design produces opposing predictions for the effects of monaural sound level and of binaural selectivity to ILD in the right-hemispheric brain responses. Selectivity to ILD would predict stronger adaptation with adaptor ILD $+10 \mathrm{~dB}$ because this coincides the ILD of the probe sound. In contrast, if the adaptation were determined by monaural sound level, one would expect the adaptation to be stronger with the $-10 \mathrm{~dB}$ adaptor ILD because this sound is louder in the contralateral left ear. The probe ILD $+30 \mathrm{~dB}$ was presented with adaptor ILDs +30 and $+10 \mathrm{~dB}$. The purpose was to compare sensitivity to ILD between a lateral $(+30$ and $+10 \mathrm{~dB})$ and a central pair $(+10$ and $-10 \mathrm{~dB})$. This also provides an additional control for monaural effects. The monaural level differences $(10 \mathrm{~dB}$ increase in the left, $10 \mathrm{~dB}$ decrease in the right) for the two conditions (ILDs of $+10 \mathrm{vs}+30 \mathrm{~dB}$ and of $-10 \mathrm{vs}+10 \mathrm{~dB}$ ) are the same. Therefore, for a monaural mechanism these stimuli should produce similar results. However, in a binaural mechanism the ILD difference across midline $(-10 \mathrm{vs}+10 \mathrm{~dB})$ is likely to produce stronger selectivity than another one with ILD differences of similar magnitude centered around a lateral ILD $(+10$ vs $+30 \mathrm{~dB})$.

MEG was recorded with a whole-head device (4D Vectorview, Elekta) at $0.01-300 \mathrm{~Hz}$ with a sampling rate of $1024 \mathrm{~Hz}$. Eye-movements and blinks were monitored with horizontal and vertical electro-oculogram (EOG). Data was filtered at $2-20 \mathrm{~Hz}$ and averaged offline from $100 \mathrm{~ms}$ before to $500 \mathrm{~ms}$ after stimulus onset. Epochs with deviations larger than $150 \mu \mathrm{V}$ in EOG or larger than $3000 \mathrm{fT} / \mathrm{cm}$ in MEG

(A)

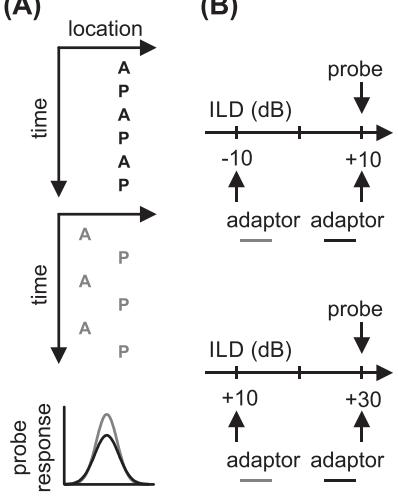

(C)
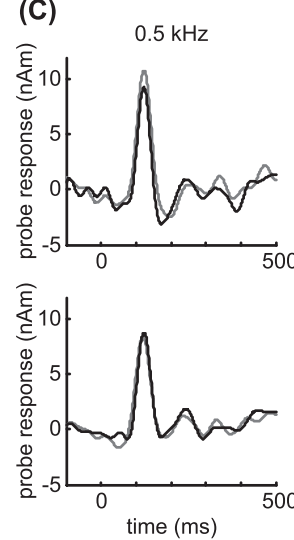

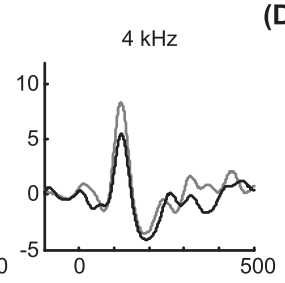

(D) $\rightarrow 0.5 \mathrm{kHz}$
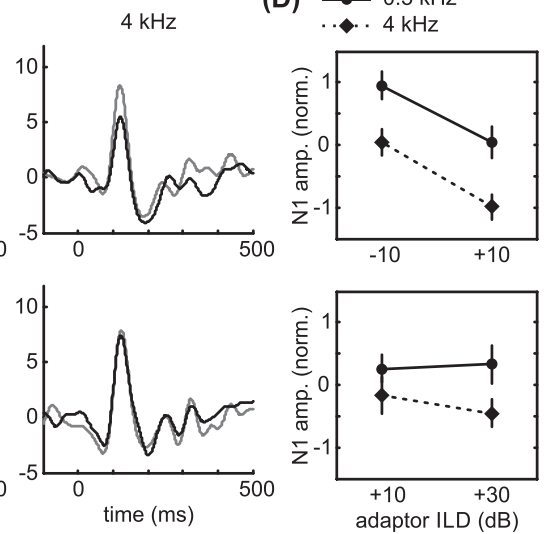

Fig. 1. (A) Probe (P) and adaptor (A) sounds were presented in a sequence of constant (black) and varying (gray) ILD. The varying sequence was expected to elicit larger response amplitudes provided that there is cortical sensitivity to ILD. (B) Two probe ILDs were used $(+10$ and +30$)$ and the adaptor ILD was the same or $20 \mathrm{~dB}$ smaller. (C) Right-hemispheric MEG responses to the probe sounds (averaged over 11 subjects) were smaller when the adaptor ILD coincided that of the probe for the $+10 \mathrm{~dB}$ probe (top) but not for the $+30 \mathrm{~dB}$ probe. (D) The average N1 amplitude (+/- standard error of the mean) was larger for the low- than the highfrequency stimulus but selectivity to ILD was similar across frequencies. 
were discarded. Approximately 100 artifact-free repetitions were acquired for each condition. To quantify the amplitude of the auditory cortical N1 response, a single equivalent current dipole (ECD) was fitted to the data from 22 gradiometer pairs covering the right temporal lobe for each subject and the two sound frequencies separately. Left-hemispheric data was not considered because the experiment was designed to disambiguate between binaural and monaural effects specifically in the right hemisphere. First, a dipole model was obtained by fitting an ECD at $1 \mathrm{~ms}$ intervals to the average of the event-related fields for the -10 and $+10 \mathrm{~dB}$ ILD stimuli and by identifying the N1 peak at the $80-150 \mathrm{~ms}$ latency. The average over these two conditions was used as the starting point because of its good signal-to-noise ratio and balance across the hemifields. The location and orientation corresponding to this peak were then used for modeling the activity elicited by the probe sounds in all conditions. From the resulting waveforms, the N1 was identified as the response peak occurring at the latency $80-150 \mathrm{~ms}$ after stimulus onset. The resulting N1 amplitudes were submitted to repeated-measures analysis of variance (stimulus frequency $\times$ adaptor ILD). For illustration [Fig. 1(D)], the N1 amplitudes were normalized separately for each participant with respect to their average and standard deviation.

\section{Results}

The N1 response amplitudes to the probe sound with ILD $+10 \mathrm{~dB}$ depended on adaptor ILD \{Figs. 1(C) and 1(D), top, main effect of adaptor ILD: $F[1,10]=44.2$, $p=0.0001\}$. The adaptation was stronger when the probe and adaptor ILDs were the same $(+10 \mathrm{~dB})$ than when the adaptor ILD differed from the probe $(-10 \mathrm{~dB})$. This is consistent with cortical selectivity to ILD but opposite to what monaural level effects would predict. Overall, the N1 amplitude was larger for the low- than the highfrequency sound (main effect of frequency: $\mathrm{F}[1,10]=4.75, p=0.05$ ). ILD sensitivity did not differ between the high- and low-frequency stimuli (interaction between frequency and adaptor ILD: $\mathrm{F}[1,10]=0.91, p=0.36$ ).

The amplitude of the N1 response to the $+30 \mathrm{~dB}$ probe did not depend on the adaptor ILD: the amplitudes were similar independent on whether the probe and the adaptor ILD differed or not (main effect of adaptor: $\mathrm{F}[1,10]=0.36, p=0.57$; Figs. $1(C)$ and 1(D), bottom $\}$. This applied to both low- and high-frequency stimuli (interaction between frequency and adaptor ILD: $\mathrm{F}[1,10]=0.02, p=0.90)$.

\section{Discussion}

The present study assessed the frequency-dependency of cortical sensitivity to ILD. The cortical activity showed selectivity to ILD that could not be explained by monaural effects. This selectivity was similar for the low- and high-frequency stimuli and was found for ILD differences crossing the midline but not for ILDs within the same hemifield.

Previous psychoacoustical studies have shown that ILD detection thresholds vary little as a function of frequency. ${ }^{3,4}$ This is consistent with the present recordings of brain activity: no significant differences were found between the two stimulus frequencies. Further, the ILD selectivity was strong when crossing midline but absent for lateral ILDs. This is also broadly in line with behavioral performance: ILD is better detected with a central than a strongly lateralized reference. ${ }^{3,4}$ However, differences in behavioral performance are relatively small compared to the complete absence of neural sensitivity in the lateral condition here. This may be due to the lateral ILD here being larger than those employed in psychoacoustics and also larger than or at the upper limit of ILDs that occur in free field. ${ }^{2}$ Further, the present measure of ILD sensitivity was quite conservative in its attempt to avoid monaural confounds. The insensitivity to lateral ILD differences may be related to the shape of spatial receptive fields in the auditory cortex. The majority of spatially sensitive neurons have wide tuning that covers an entire hemifield of auditory space. ${ }^{7,8}$ Such neurons are sensitive to location differences near midline but their activity level may saturate for lateral locations. 
The lack of strong neural and perceptual specialization to high-frequency ILD may seem surprising considering the spatial cues available in free field. Prominent ILDs occur only for high sound frequencies. ${ }^{2}$ However, sound sources very near to the head, i.e., at a distance less than $1 \mathrm{~m}$ can lead to ILDs of $10 \mathrm{~dB}$ and beyond at around $500 \mathrm{~Hz}$ (the center frequency of the present low-frequency stimulus). ${ }^{9}$ The neural and perceptual sensitivity to low-frequency ILD may therefore serve binaural processing in conditions where sound sources are near to the head.

Even though ILD perception does not depend strongly on frequency in terms of detection thresholds, the extent of perceived laterality can be larger for high- than low-frequency stimuli. ${ }^{10}$ This suggests that some specialization to ILD in higher frequencies exists even though it did not show in the cortical ILD-selectivity measured here. The neural measures of selectivity developed here may be sensitive to detection and discrimination mechanisms rather than those underlying the perception of laterality. Future work will need to explore alternative measures of cortical ILD sensitivity and this may reveal additional neural mechanisms of ILD processing, possibly frequency-dependent ones, that the present method was not sensitive to.

\section{Acknowledgments}

I thank J. Lamminsalo for assistance. This study was supported by the Academy of Finland (Grant No. 13257844).

\section{References and links}

${ }^{1}$ J. C. Middlebrooks and D. M. Green, "Sound localization by human listeners," Annu. Rev. Psychol. 42, 135-159 (1991).

${ }^{2}$ E. A. G. Shaw, "Transformation of sound pressure level from the free field to the eardrum in the horizontal plane,” J. Acoust. Soc. Am. 56, 1848-1861 (1974).

${ }^{3}$ W. A. Yost and R. H. Dye, "Discrimination of interaural differences of level as a function of frequency," J. Acoust. Soc. Am. 83, 1846-1851 (1988).

${ }^{4}$ E. R. Hafter, R. H. Dye, J. M. Neutzel, and H. Aronow, "Difference thresholds for interaural intensity," J. Acoust. Soc. Am. 61, 829-834 (1977).

${ }^{5}$ J. P. Mäkelä and L. McEvoy, “Auditory evoked fields to illusory sound source movements," Exp. Brain Res. 110, 446-454 (1996).

${ }^{6}$ H. C. Hart, A. R. Palmer, and D. A. Hall, "Different areas of human non-primary auditory cortex are activated by sounds with spatial and nonspatial properties,” Hum. Brain Mapp. 21, 178-190 (2004).

${ }^{7}$ N. H. Salminen, P. J. May, P. Alku, and H. Tiitinen, "A population rate code of auditory space in the human cortex," PLoS One 4, e7600 (2009).

${ }^{8}$ G. C. Stecker, I. A. Harrington, and J. C. Middlebrooks, "Location coding by opponent neural populations in the auditory cortex," PLoS Biol. 3, e78 (2005).

${ }^{9}$ D. S. Brungart and W. M. Rabinowitz, "Auditory localization of nearby sources. Head-related transfer functions," J. Acoust. Soc. Am. 106, 1465-1479 (1999).

${ }^{10}$ L. R. Bernstein and C. Trahiotis, "Lateralization produced by interaural intensitive disparities appears to be larger for high-vs low-frequency stimuli," J. Acoust. Soc. Am.129, EL15-EL20 (2011). 